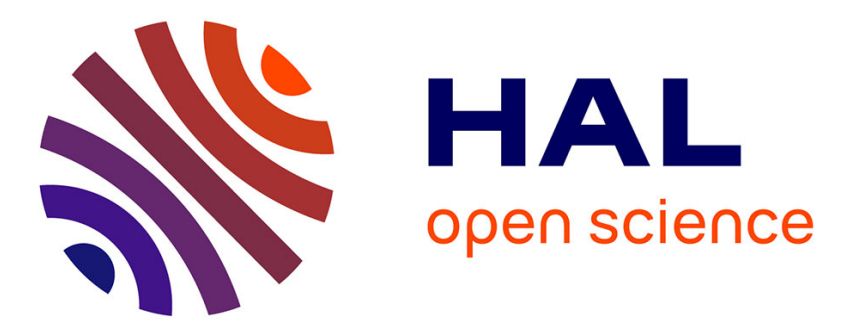

\title{
A new device to restore sensory congruency in virtual reality and to prevent cybersickness
}

\author{
Estelle Nakul, Renaud Jeannin, Christophe Lopez
}

\section{To cite this version:}

Estelle Nakul, Renaud Jeannin, Christophe Lopez. A new device to restore sensory congruency in virtual reality and to prevent cybersickness. 2021 IEEE Conference on Virtual Reality and 3D User Interfaces Abstracts and Workshops (VRW), 2021, 10.1109/vrw52623.2021.00036 . hal-03282846

\section{HAL Id: hal-03282846 \\ https://hal-amu.archives-ouvertes.fr/hal-03282846}

Submitted on 9 Jul 2021

HAL is a multi-disciplinary open access archive for the deposit and dissemination of scientific research documents, whether they are published or not. The documents may come from teaching and research institutions in France or abroad, or from public or private research centers.
L'archive ouverte pluridisciplinaire HAL, est destinée au dépôt et à la diffusion de documents scientifiques de niveau recherche, publiés ou non, émanant des établissements d'enseignement et de recherche français ou étrangers, des laboratoires publics ou privés. 


\title{
A new device to restore sensory congruency in virtual reality and to prevent cybersickness
}

\author{
Estelle Nakul ${ }^{1,2 *}$, Renaud Jeannin ${ }^{2}$, Christophe Lopez ${ }^{1}$ \\ 1: Aix Marseille Univ, CNRS, LNC, FR3C, Marseille, France \\ 2: Boarding Ring, Ollioules, France
}

\begin{abstract}
Cybersickness likely originates from contradictory sensory signals about user's motion in virtual reality. Most proposed solutions reduce visual inputs to decrease vection intensity or the sensory conflict. In contrast with this approach the Seenetic VR, inserted into the head-mounted display, adds information in the peripheral visual field congruent with self-motion signals. We present this new technology to prevent cybersickness and the research protocols to quantify its effects and contribute to a better understanding of the sensory mechanisms of cybersickness.
\end{abstract}

Keywords: Cybersickness, peripheral visual information, vection, Seenetic VR.

Index Terms: Human-centered computing - Human Computer Interaction (HCI) - Virtual Reality - Perception

\section{INTRODUCTION}

Virtual reality (VR) is increasingly used for entertainment, research, education and training, as well as for therapies. However, VR can cause symptoms similar to those of motion sickness. This "virtual reality sickness", referred to as cybersickness, affects $45 \%$ of users and may be the main obstacle to the deployment of VR [1].

Cybersickness is characterized by feelings of body heat, sweating and cold sweats, drowsiness, increased salivation, gastric sensations, nausea, vomiting, dizziness, sometimes headaches, loss of appetite and increased sensitivity to odors [2]. Cybersickness most often occurs during exposure to a moving virtual environment with little or no user's movement. These effects dissipate when immersion in VR ends, but they can last for several hours. There is therefore a need to develop efficient and easy to use solutions to overcome cybersickness and harness the full potential of VR.

Cybersickness is mostly considered to originate from a sensory conflict. Like motion sickness, cybersickness may come from a conflict between visual information and vestibular information from the inner ear [1], [2]. The mechanisms linking multisensory conflict and cybersickness are still being studied, but aggravating factors have been identified [1]. These can be technical limitations such as lag between user commands and their actual occurrence in VR, position tacking errors and image jitter. Sex, fatigue, anxiety and illness (e.g. flu) may also worsen user experience in VR [1].

An optical flow can create an illusory sensation of self-motion called vection [3]-[5]. Vection is thought to facilitate cybersickness [6] : when a static user is exposed to visual motion, it conflicts with other senses such as the vestibular system, which indicates that the body is stationary. In the case of a sensory conflict, vection intensity in VR could be an indicator of the onset of cybersickness [7], [8].

*estelle.nakul@univ-amu.fr
Another theory posits that postural instability favors cybersickness [9], [10]. Several studies showed that individuals with poorer postural control measured before exposure to VR are more sensitive to visual simulation of self-movement [10], [11]. Studies also found that restraining head and trunk motion reduced cybersickness in older adults [12]. Thus, spontaneous postural instability should predict susceptibility to cybersickness, and its onset should be preceded by an increase in postural instability.

Current commercially available solutions for motion sickness and cybersickness are generally drug-based, have side effects, and are intended to treat the consequences of cybersickness (e.g., nausea) rather than to prevent it. Head mounted displays (HMDs) are also being improved to support higher framerates and reduce phase lag between actual and virtual motion that can negatively impact the user experience. Alternative approaches focus on the software by adding stable reference frames in the virtual environment, image blurring, or field of view reduction [13]. These aim at decreasing or removing peripheral visual information and may reduce vection, which requires a large field of view [3], [5]. In all cases the idea is to prevent peripheral optical flow from contributing to sensory conflicts. However, the effectiveness of these approaches is still debated [13], [14], and reducing the field of view may degrade user experience to the point that some users prefer not to benefit from these techniques [14]. In addition, these software adaptations are not always compatible with the VR environments, although efforts are beginning to be made in this direction [13].

Thus, most current solutions propose to reduce visual signals to reduce vection intensity or the visuo-vestibular conflict. In contrast to this approach, we present a new device with a novel approach to reduce VR-induced visuo-vestibular conflicts. Instead of reducing the field of view, the Seenetic VR device adds inertial movement signals in the peripheral visual field that are congruent with the user's head movements. It restores the congruency of visual information with vestibular, somatosensory and motor efferent copy signals when the user moves while immersed in VR.

\section{The Seenetic VR deVice}

The Seenetic VR (Boarding Ring, Ollioules, France) is an addon device providing inertial cues in the peripheral visual field of the user in an HMD, consistent with the movements of the user's head and independent of the virtual environment. This electronic device introduces, in the user's lateral peripheral visual field, a grid of nine dots reporting inertial and static information about the user's head movements and position relatively to gravity. The dots compensate for the movements of the user's head, by moving in the opposite direction as the user's movements.

The Seenetic VR device consists of two displays located in the HMD on the right side of the right eye and on the left side of the left eye respectively, an inertial measurement unit located behind the right display, a microcontroller, a power supply system (battery or direct current via micro-USB) and a holding device. The displays are placed in the peripheral visual field, inside the $\mathrm{HMD}$, as close as possible to the screens, without obstructing all 
or part of the main image. Its holding system allows the device to be compatible with several HMDs.

The Seenetic VR weights 20 g equally distributed on both sides of the HMD. Increasing the weight and antero-posterior imbalance of the HMD by at least $100 \mathrm{~g}$ can reduce the user experience quality in VR [15]. However, the Seenetic VR low weight avoids such imbalance effects.
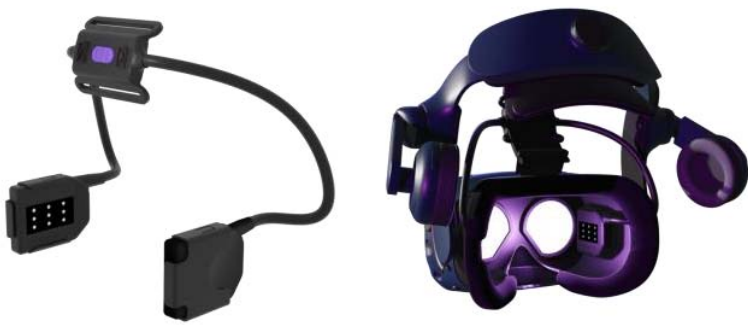

Figure 1: On the left, the Seenetic VR device, on the right, the Seenetic VR mounted on an HTC Vive Pro.

The displays take the form of two 1.3-inch OLED screens (resolution: $64 \times 128$ pixels; refresh rate: $90 \mathrm{~Hz}$ ) inserted on the side walls of the HMD to deliver peripheral visual stimuli. Visual stimuli consist of nine light dots delivered with a light intensity of 25 Lux. When there is no user's head movement the dots are static. After $3 \mathrm{~s}$ without head motion, the dots start to flicker until there is movement again.

When the user moves her head, the refresh rate of $90 \mathrm{~Hz}$ allows the dots to move with a latency under $10 \mathrm{~ms}$, which is well below the mean perceptual threshold of $148 \mathrm{~ms}$ for lag detection in HMDs [16]. The movement of the dots corresponds to the inverse of the angular accelerations of the head recorded by the inertial measurement unit. This provides peripheral visual information consistent with vestibular information and other signals related to head motion (such as proprioceptive signals from the neck muscles). These visual signals reproduce a movement of the image on the retina during a head motion in the real environment.

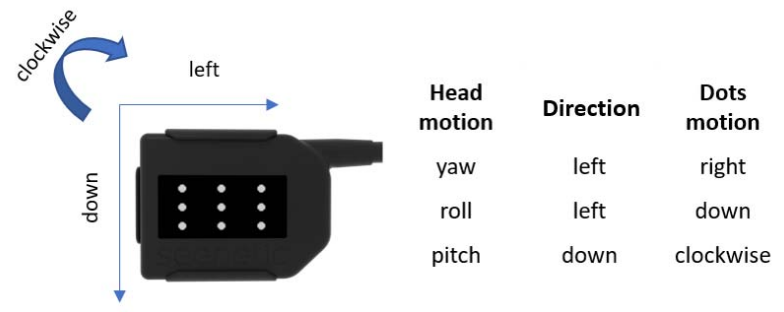

Figure 2: Visual stimulation delivered by the Seenetic VR device from the right-side display.

\section{EXPERIMENTS AND DISCUSSION}

\subsection{Seenetic VR effects on cybersickness}

Initial informal tests and user feedback are positive about the effectiveness of the device. We designed two experiments to quantify how manipulating visuo-vestibular congruency using the Seenetic VR modulates cybersickness, as measured subjectively by questionnaires and objectively by electrophysiological recordings.

In a first experiment, thirty participants will be seated and wear an HMD equipped with the Seenetic VR device. We will include a similar proportion of male and female participants to analyze potential sex differences in the sensitivity to motion sickness [11], [17]. They will be immersed in ten-minute virtual roller coaster scenarios, which are frequently used to induce and study cybersickness [18], [19]. Participants will be prompted to move their heads in all directions to explore the VR environment during the roller coaster ride, after which they will have to answer questions about visual elements of the scenes they visited. The Seenetic VR will work in different modes to create different experimental conditions. In the "off" condition, the device will be switched off and will therefore not deliver any peripheral visual stimulation. When it will be turned on, dots will appear on both screens, on each side of the participants' heads. In the "on" condition, the Seenetic VR will function normally: the dots will move according to the movements of the participants' heads to provide visual peripheral movement information congruent with head motion. A "sham" mode was also developed in order to compare the effect of peripheral visual information related or unrelated to the user's head motion. This aims at controlling potential distracting effects of peripheral visual signals on cybersickness. In this mode, three random angles are generated to simulate the pitch, roll and yaw motion. A filter smooths the generated motion so that for each frame displayed, the calculation is based on angles that are $0.1 \times$ the random angle $+0.9 \times$ the actual angle. The number of frames before the generation of a new angle was fixed at 150, 200 and 250 for the pitch, roll and yaw respectively. As the device refresh rate is around $90 \mathrm{~Hz}$, the resulting motion simulated lasts about $1.7 \mathrm{~s}, 2.2 \mathrm{~s}$ and $2.8 \mathrm{~s}$ before the generation of new angles. The maximum value for each angle was set to $180^{\circ}$.

Each condition ("on", "off", "sham") will be presented once in a random order, during two sessions that will take place one week apart on the same day of the week and at the same time of the day. Half of the participants will be exposed to the "on" and "off" conditions (group A), while the other half will be exposed to the "on" and "sham" conditions (group B). Participants will be attributed to group A or to group B pseudo-randomly, so that there will be equal numbers of women and men in each group.

Three subjective measures of cybersickness will be used. The Simulator Sickness Questionnaire (SSQ; [20]) is one of the most widely used cybersickness questionnaires. The SSQ scores overall cybersickness, as well as three categories of symptoms (oculomotor symptoms, disorientation and nausea). Participants will complete the SSQ once at the beginning of each session to obtain pre-exposure scores, and once after each ten-minute exposure to the virtual roller coaster (post-exposure scores). Every two minutes during exposure to the virtual roller coaster, participants will also indicate their level of cybersickness using the 11-point misery scale (MISC; [21], [22]) and a forced choice question about motion sickness. The MISC is simple and quick and can be applied repeatedly in VR without interrupting immersion. This will provide a measure of cybersickness intensity throughout the ride on the virtual roller coaster. Participants will also have to answer "yes" or "no" to the question "Are you motion sick?".

The SSQ and MISC scores and the answer to the forced choice question will be compared between the conditions "on", "off" and "sham". Post-exposure SSQ scores for each condition will be compared to their respective pre-exposure scores. Although administrating the SSQ before and after exposure to VR may increase SSQ scores [23], this effect should be present in both sessions, making it possible to compare SSQ scores between sessions. Based on the hypothesis of a visuo-vestibular conflict, providing lateral peripheral visual information congruent with vestibular information should reduce cybersickness. We expect the MISC and SSQ scores during the "on" condition to be 
significantly lower than the scores during the "off" and "sham" conditions. We will also compare the latency at which participants indicate that they feel motion sick when answering the forced choice question. We expect that participants will report no motion sickness or report it later during exposure to the virtual roller coaster for the "on" condition compared to "off" and "sham" conditions.

In addition, we will use two measures of cybersickness based on non-invasive electrophysiological recordings. Cybersickness is typically accompanied by an increase in sweating and a decrease in body temperature [2], [24]. We will measure forehead skin conductance, which reflects the activity of the sweat glands, and skin temperature in the left hand throughout the different conditions to which participants will be exposed. We expect skin conductance to increase during exposure to the virtual roller coaster for all conditions. The increase is expected to be lower for the "on" condition compared to the "off" and "sham" conditions. We expect the skin temperature to decrease during the exposure to the virtual roller coaster and this decrease to be lower for the "on" condition compared to the "off" and "sham" conditions.

Vection intensity will also be evaluated once after exposure to the virtual roller coaster using a visual analog scale on which participants will be asked to indicate the strength of the illusion of movement they felt during the ride. Participants reporting higher intensities of vection should more easily or quickly experience symptoms associated with cybersickness [7], [8].

A questionnaire will also allow participants to provide free feedback on the Seenetic VR device. We hypothesize that the Seenetic VR will reduce the symptoms associated with cybersickness without users reporting any discomfort regarding the presence of the device in the HMD.

\subsection{Seenetic VR effects and postural control}

In a second experiment, we will use predictions from the postural instability theory, which posits that spontaneous postural instability facilitates cybersickness, to test the efficiency of the Seenetic VR to reduce cybersickness [10], [14]. Thirty participants, different from those of the first experiment, will be included. As in the first experiment, we will recruit a similar proportion of males and females to analyze potential sex differences in the sensitivity to motion sickness [11], [17]. Before any VR exposure, we will measure postural stability using a force platform with three strain gauges to calculate the displacements of the participants' center of pressure in the horizontal plane. Participants will be required to stand on the platform for four oneminute sessions, one with their eyes open, another one with their eyes closed and then two other sessions with visual inspection and search tasks (following procedures described in [11]). After that, participants will also be asked to stand on the force platform while exposed to the roller coasters for 10 minutes, in three different conditions depending on the Seenetic VR mode ("on", "off", "sham"). We will analyze classical indices of total length, area and velocity as well as more sophisticated ones such as the wavelet transform and the multifractal spectrum of center of pressure displacements [25], [11]. These indices will be used to compare postural stability between participants as well as the effect of each conditions ("on", "off" and "sham"). According to the postural instability hypothesis, participants in which center of pressure displacement indices will indicate a poorer postural stability should be more prone to cybersickness than more stable participants. We should also observe an increase in postural instability during VR exposures evoking cybersickness. We hypothesize that postural instability will be reduced in the "on" condition compared to the "sham" condition.
Cybersickness will be measured as in the first experiment. Participants will complete the SSQ once before any exposure to VR (pre-exposure scores) and once after each ten-minute exposure to the virtual roller coaster (post-exposure scores). They will also answer to the MISC and the forced choice question every two minutes during their exposure to the roller coaster, as described for the first experiment. These cybersickness measures will be compared between the conditions "on", "off" and "sham". Post-exposure SSQ scores for each condition will also be compared to their respective pre-exposure scores. We expect to confirm results from subjective measures of cybersickness from the first to the second experiment. Less stable participants should show higher SSQ and MISC scores than more stable participants. We also hypothesize that the latency for participants to answer "yes" to the forced choice motion sickness question may be linked with an increase in postural instability during exposure to the virtual roller coaster.

Results from this experiment will inform whether predictions from the postural instability theory may help to assess the efficiency of interventions against cybersickness. It may also determine whether the Seenetic VR has different effects depending on individual spontaneous postural stability.

\subsection{Traits influencing cybersickness}

We will also consider individual factors that may affect cybersickness. Once at the beginning of the experiment and prior to any exposure to VR, participants will complete the Motion Sickness Susceptibility Questionnaire (MSSQ; adapted from [26], [27]). The MSSQ assesses susceptibility to motion sickness and will allow to measure its impact on the potential effects of the Seenetic VR. Moreover, symptoms of cybersickness resemble physiological manifestations that come with anxiety, which has been shown to increase SSQ scores [28]. A higher level of selfreported anxiety could therefore increase cybersickness during VR exposure, or at least worsen the measurement of these symptoms by the SSQ. Once at the beginning of each experiment, participants will complete the French version of the State-Trait Anxiety Inventory (STAI-Y; [29], [30]) in order to assess their anxiety as a personality trait and as an emotional state related to the current situation. Participants' anxiety scores will be correlated with their cybersickness scores to test for a possible relationship between anxiety and these measures. If no correlation is observed, we will have controlled for the fact that this psychological aspect of the participants' condition does not affect the other possible effects observed. If a correlation is observed, we will be able to analyze this relationship between anxiety and cybersickness and bring new elements to current research on this topic.

Finally, sex effects on cybersickness, with female users being more sensitive to it than males, are still controversial [11], [17]. Studies suggest that females might be more prone to cybersickness due to poorer postural stability [11]. We will therefore compare subjective and objective cybersickness measures as well as MSSQ scores, anxiety scores and spontaneous postural stability between male and female participants to test potential sex effects and their relation to the effect of the Seenetic VR. The results of this research will therefore contribute to the advancement of fundamental knowledge on the multisensory mechanisms underlying cybersickness. They will also contribute to testing the Seenetic VR device, an innovative and non-invasive solution to mitigate cybersickness, aimed at professionals and the general public alike. 


\section{ACKNOWLEDGMENTS}

This work has received support from the French government under the Programme "Investissements d'Avenir", Initiative d'Excellence d'Aix-Marseille Université via AMidex funding (AMX-19-IET-004), and ANR (ANR-17-EURE-0029). The authors thank NeuroMarseille and the NeuroSchool for their support.

\section{References}

[1] J. J. LaViola, "A discussion of cybersickness in virtual environments," ACM SIGCHI Bull., vol. 32, no. 1, pp. 4756, Jan. 2000, doi: 10.1145/333329.333344.

[2] J. F. Golding, "Motion sickness," in Handbook of Clinical Neurology, vol. 137, J. M. Furman and T. Lempert, Eds. Elsevier, 2016, pp. 371-390.

[3] T. Brandt, J. Dichgans, and E. Koenig, "Differential effects of central verses peripheral vision on egocentric and exocentric motion perception," Exp. Brain Res., vol. 16, no. 5, pp. 476-491, Mar. 1973, doi: 10.1007/BF00234474.

[4] A. Berthoz, B. Pavard, and L. R. Young, "Perception of linear horizontal self-motion induced by peripheral vision (linearvection) basic characteristics and visual-vestibular interactions," Exp. Brain Res., vol. 23, no. 5, pp. 471-489, Nov. 1975, doi: 10.1007/BF00234916.

[5] S. Palmisano, R. S. Allison, M. M. Schira, and R. J. Barry, "Future challenges for vection research: definitions, functional significance, measures, and neural bases," Front. Psychol., vol. 6, 2015, doi: 10.3389/fpsyg.2015.00193.

[6] B. Keshavarz, B. E. Riecke, L. J. Hettinger, and J. L. Campos, "Vection and visually induced motion sickness: how are they related?," Front. Psychol., vol. 6, p. 472, 2015, doi: 10.3389/fpsyg.2015.00472.

[7] F. Bonato, A. Bubka, S. Palmisano, D. Phillip, and G. Moreno, "Vection change exacerbates simulator sickness in virtual environments," Presence Teleoperators Virtual Environ., vol. 17, no. 3, pp. 283-292, Jun. 2008, doi: 10.1162/pres.17.3.283.

[8] S. A. E. Nooij, P. Pretto, D. Oberfeld, H. Hecht, and H. H. Bülthoff, "Vection is the main contributor to motion sickness induced by visual yaw rotation: Implications for conflict and eye movement theories," PloS One, vol. 12, no. 4, p. e0175305, 2017, doi: 10.1371/journal.pone.0175305.

[9] G. E. Riccio and T. A. Stoffregen, "An ecological theory of motion sickness and postural instability," Ecol. Psychol., vol. 3, no. 3, pp. 195-240, 1991, doi: 10.1207/s15326969eco0303_2.

[10] T. A. Stoffregen and L. J. Smart, "Postural instability precedes motion sickness," Brain Res. Bull., vol. 47, no. 5, pp. 437-448, Nov. 1998, doi: 10.1016/s03619230(98)00102-6.

[11] J. Munafo, M. Diedrick, and T. A. Stoffregen, "The virtual reality head-mounted display Oculus Rift induces motion sickness and is sexist in its effects," Exp. Brain Res., vol. 235, no. 3, pp. 889-901, 2016, doi: 10.1007/s00221-0164846-7.

[12] B. Keshavarz, A. C. Novak, L. J. Hettinger, T. A. Stoffregen, and J. L. Campos, "Passive restraint reduces visually induced motion sickness in older adults," J. Exp. Psychol. Appl., vol. 23, no. 1, pp. 85-99, 2017, doi: 10.1037/xap0000107.

[13] S. Ang and J. Quarles, "GingerVR: An Open Source Repository of Cybersickness Reduction Techniques for Unity," in 2020 IEEE Conference on Virtual Reality and 3D
User Interfaces Abstracts and Workshops (VRW), Mar. 2020, pp. 460-463, doi: 10.1109/VRW50115.2020.00097.

[14] J. Teixeira and S. Palmisano, "Effects of dynamic field-ofview restriction on cybersickness and presence in HMDbased virtual reality," Virtual Real., Aug. 2020, doi: 10.1007/s10055-020-00466-2.

[15] K. Ito, M. Tada, H. Ujike, and K. Hyodo, "Effects of Weight and Balance of Head Mounted Display on Physical Load," 2019, pp. 450-460.

[16] J. D. Moss, E. R. Muth, R. A. Tyrrell, and B. R. Stephens, "Perceptual thresholds for display lag in a real visual environment are not affected by field of view or psychophysical technique," Displays, vol. 31, no. 3, pp. 143149, Jul. 2010, doi: 10.1016/j.displa.2010.04.002.

[17] S. Grassini and K. Laumann, "Are Modern Head-Mounted Displays Sexist? A Systematic Review on Gender Differences in HMD-Mediated Virtual Reality," Front. Psychol., vol. 11, Aug. 2020, doi: 10.3389/fpsyg.2020.01604.

[18] A. M. Gavgani, D. M. Hodgson, and E. Nalivaiko, "Effects of visual flow direction on signs and symptoms of cybersickness," PLOS ONE, vol. 12, no. 8, p. e0182790, Aug. 2017, doi: 10.1371/journal.pone.0182790.

[19] R. Islam, Y. Lee, M. Jaloli, I. Muhammad, D. Zhu, and J. Quarles, Automatic Detection of Cybersickness from Physiological Signal in a Virtual Roller Coaster Simulation. 2020.

[20] R. S. Kennedy, N. E. Lane, K. S. Berbaum, and M. G. Lilienthal, "Simulator Sickness Questionnaire: An Enhanced Method for Quantifying Simulator Sickness," Int. J. Aviat. Psychol., vol. 3, no. 3, pp. 203-220, Jul. 1993, doi: 10.1207/s15327108ijap0303_3.

[21] P. J. Feenstra, J. E. Bos, and R. N. H. W. van Gent, "A visual display enhancing comfort by counteracting airsickness," Displays, vol. 32, no. 4, pp. 194-200, Oct. 2011, doi: 10.1016/j.displa.2010.11.002.

[22] A. K. T. Ng, L. K. Y. Chan, and H. Y. K. Lau, "A study of cybersickness and sensory conflict theory using a motioncoupled virtual reality system," Displays, vol. 61, p. 101922, Jan. 2020, doi: 10.1016/j.displa.2019.08.004.

[23] S. Young, B. Adelstein, and S. Ellis, "Demand characteristics in assessing motion sickness in a virtual environment: or does taking a motion sickness questionnaire make you sick?," IEEE Trans. Vis. Comput. Graph., vol. 13, pp. 422-8, Jan. 2007.

[24] Y. Y. Kim, H. J. Kim, E. N. Kim, H. D. Ko, and H. T. Kim, "Characteristic changes in the physiological components of cybersickness," Psychophysiology, vol. 42, no. 5, pp. 616625, Sep. 2005, doi: 10.1111/j.1469-8986.2005.00349.x.

[25] L. R. Young, L. Bernard-Demanze, M. Dumitrescu, J. Magnan, L. Borel, and M. Lacour, "Postural performance of vestibular loss patients under increased postural threat," $J$. Vestib. Res., vol. 22, no. 2,3, pp. 129-138, Jan. 2012, doi: 10.3233/VES-2012-0449.

[26] J. F. Golding, "Predicting individual differences in motion sickness susceptibility by questionnaire," Personal. Individ. Differ., vol. 41, no. 2, pp. 237-248, Jul. 2006, doi: 10.1016/j.paid.2006.01.012.

[27] A. C. Paillard et al., "Motion sickness susceptibility in healthy subjects and vestibular patients: effects of gender, age and trait-anxiety," J. Vestib. Res. Equilib. Orientat., vol. 23, no. 4-5, pp. 203-209, 2013, doi: 10.3233/VES-130501.

[28] Y. Ling, W. P. Brinkman, H. T. Nefs, C. Qu, and I. E. J. Heynderickx, "Cybersickness and anxiety in virtual 
environments," 2011, Accessed: Jan. 18, 2021. [Online]. Available:

https://research.tue.nl/en/publications/cybersickness-andanxiety-in-virtual-environments.

[29] C. Spielberger, Manual for the State-Trait Anxiety Inventory (Form Y)., Palo Alto, California: Consulting Psychologists Press. 1983.

[30] J. Gauthier and S. Bouchard, "Adaptation canadiennefrançaise de la forme révisée du State-Trait Anxiety Inventory de Spielberger [A French-Canadian adaptation of the revised version of Spielberger's State-Trait Anxiety Inventory]," Can. J. Behav. Sci. Rev. Can. Sci. Comport., vol. 25, no. 4, pp. 559-578, 1993. 